\title{
Mixed Th2 and non-Th2 inflammatory pattern in the asthma-COPD overlap: a network approach
}

This article was published in the following Dove Press journal:

International Journal of COPD

Luis Pérez de Llano, ${ }^{1, *}$ Borja G Cosío, ${ }^{2,3, *}$ Amanda Iglesias, ${ }^{3}$ Natividad de las Cuevas, ${ }^{4}$ Juan Jose Soler-Cataluña, ${ }^{3,5}$ Jose Luis Izquierdo, 6 Jose Luis López-Campos, ${ }^{3,7}$ Carmen Calero, ${ }^{7}$ Vicente Plaza, ${ }^{3,8-10}$ Marc Miravitlles, ${ }^{3,11}$ Alfons Torrego, ${ }^{8-10}$ Eva Martinez-Moragon, ${ }^{12}$ Joan B Soriano, ${ }^{13}$ Antolin Lopez Viña, ${ }^{14}$ Irina Bobolea ${ }^{15}$

\section{On behalf of the CHACOS} Study Group

'Department of Respiratory Medicine, Hospital Lucus Augusti, Lugo, Spain; ${ }^{2}$ Department of Respiratory Medicine, Hospital Universitario Son Espases-IdISBa, Palma de Mallorca, Spain ${ }^{3}$ CIBER de Enfermedades Respiratorias, Instituto de Salud Carlos III, Madrid, Spain;

${ }^{4}$ Department of Allergy, Hospital 12 de Octubre, Madrid, Spain; ${ }^{5}$ Department of Respiratory Medicine, Hospital Arnau de Vilanova, Valencia, Spain; ${ }^{6}$ Department of Respiratory Medicine, Hospital Universitario de Guadalajara, Guadalajara, Spain;

${ }^{7}$ Department of Respiratory Medicine, Hospital Virgen del Rocío, Sevilla, Spain; ${ }^{8}$ Department of Respiratory Medicine, Hospital de la Santa Creu y Sant Pau, Barcelona, Spain; ' Institut d'Investigació Biomédica Sant Pau, IIB Sant Pau, Barcelona, Spain; ${ }^{10}$ Department of Medicine, Universitat Autònoma de Barcelona, Barcelona, Spain; "Department of Respiratory Medicine, Hospital Universitari Vall d'Hebron, Barcelona, Spain; ${ }^{12}$ Department of Respiratory Medicine, Hospital Dr Peset, Valencia, Spain; ${ }^{13}$ Instituto de Investigación Hospital de la Princesa, Universidad Autónoma de Madrid, Madrid, Spain; ${ }^{14}$ Department of Respiratory Medicine. Hospital Puerta de Hierro, Madrid, Spain;

${ }^{15}$ Servei de Pneumologia i Alergia, Hospital

Clinic, Barcelona, Spain

*These authors contributed equally to this work

Correspondence: Borja G Cosío Department of Respiratory Medicine, Hospital Universitario Son Espases, Carretera de Valldemossa 79, 07010 Palma de Mallorca, Spain Email borja.cosio@ssib.es
Introduction: The asthma-chronic obstructive pulmonary disease (COPD) overlap (ACO) is a clinical condition that combines features of those two diseases, and that is difficult to define due to the lack of understanding of the underlying mechanisms. Determining systemic mediators may help clarify the nature of inflammation in patients with ACO.

Objectives: We aimed at investigating the role and interaction of common markers of systemic inflammation (IL-6, IL-8, and tumor necrosis factor- $\alpha$ ), Th2-related markers (periostin, IL-5, and IL-13), and IL-17 in asthma, COPD, and ACO.

Methods: This is a cross-sectional study of patients aged $\geq 40$ years with a post-bronchodilator forced expiratory volume in the first second/forced vital capacity $<0.70$ recruited from outpatient clinics in tertiary hospitals with a clinical diagnosis of asthma, COPD, or ACO. ACO was defined by a history of smoking $>10$ pack-years in a patient with a previous diagnosis of asthma or by the presence of eosinophilia in a patient with a previous diagnosis of COPD. Clinical, functional, and inflammatory parameters were compared between categories using discriminant and network analysis.

Results: In total, 109 ACO, 89 COPD, and 94 asthma patients were included. Serum levels (median [interquartile range]) of IL-5 were higher in asthma patients than in COPD patients (2.09 [0.61-3.57] vs 1.11 [0.12-2.42] pg/mL, respectively; $p=0.03$ ), and IL-8 levels (median [interquartile range]) were higher in COPD patients than in asthma patients (9.45 [6.61-13.12] vs 7.03 [4.69-10.44] pg/mL, respectively; $p<0.001$ ). Their values in ACO were intermediate between those in asthma and in COPD. Principal component and network analysis showed a mixed inflammatory pattern in ACO in between asthma and COPD. IL-13 was the most connected node in the network, with different weights among the three conditions.

Conclusion: Asthma and COPD are two different inflammatory conditions that may overlap in some patients, leading to a mixed inflammatory pattern. IL-13 could be central to the regulation of inflammation in these conditions.

Keywords: asthma mechanisms, COPD mechanisms, inflammatory cytokines, overlap, network analysis, IL-13

\section{Introduction}

Asthma and chronic obstructive pulmonary disease (COPD) are common chronic inflammatory airway diseases with different underlying mechanisms. ${ }^{1}$ However, they are characterized by a complex heterogeneity and the existence of different overlapping phenotypes that influence the response to treatment. As a result, it is sometimes difficult to determine whether a patient is suffering from asthma, COPD, or the overlap of both the conditions. This controversy led to a theory - the so-called "Dutch hypothesis" - that postulates that chronic obstructive airway disease (COAD) may occur on a continuum, with "pure" forms of asthma and COPD representing 
the extremes of the same disease and the various forms of COAD being different expressions of a single disease entity. ${ }^{2}$ To make things even more complicated, clinicians have long acknowledged the existence of a group of patients with COAD who display characteristics of both asthma and COPD. This condition has been recently regaining attention in the medical literature, and it has ended up being named ACO (asthma-COPD overlap). ${ }^{3,4}$ ACO is an umbrella term that encompasses patients with COPD and eosinophilic inflammation and smoking asthmatics with a predominantly neutrophilic inflammation and irreversible airway obstruction. Therefore, the whole picture of COAD is blurred, with different and overlapping phenotypes that are difficult to clinically distinguish from one to another with the lack of an accurate biomarker that is able to distinguish these entities. We have recently shown that the presence of a Th2 signature, defined by eosinophils in sputum $>3 \%$ or blood eosinophils $>300$ cells $/ \mu \mathrm{L}$, could help to identify and treat the patients with COAD. ${ }^{5}$

In a clinical scenario of great heterogeneity, breaking down complex data into its component parts and plotting them to show their interdependences and interrelationships would help us to clarify the motley nature of COAD. The aim of this study was to investigate the systemic "molecular fingerprinting" of the different clinical phenotypes of COAD. In doing so, the main objective was to evaluate the heterogeneity of inflammation in patients with COAD by using discriminant and network analysis. The comparison of correlation networks can provide new insights into the complexity and heterogeneity of ACO. As secondary end points, we assessed the capacity of these biomarkers to predict the clinical COAD phenotype, the Th2-high pattern, and the relation between these biomarkers and clinical significant outcomes: exacerbations and symptoms.

\section{Materials and methods}

\section{Study design}

This cross-sectional, observational, multicenter study was carried out in 23 outpatient clinics from tertiary hospitals in Spain run by expert respiratory physicians. The design of the study has been described elsewhere. ${ }^{5}$ As described in detail previously, ${ }^{5}$ "male and female patients aged $\geq 40$ years with chronic airflow obstruction (post-bronchodilator forced expiratory volume in the first second $\left(\mathrm{FEV}_{1}\right) /$ forced vital capacity $(\mathrm{FVC})<0.70$ ) and a history of physiciandiagnosed asthma, COPD, or ACO (as defined below) who signed an informed consent form were included. Patients had to be in a stable condition and free from exacerbations for at least 3 months. Exclusion criteria included primary bronchiectasis, active cancer, other chronic inflammatory diseases, and poor performance status (defined as life expectancy of $<3$ months)."

A blood sample was obtained to determine the number and percentage of blood eosinophils and quantification of immunoglobulin E, and serum was stored at $-80^{\circ} \mathrm{C}$ for later determination of periostin and cytokines. The degree of compliance with inhalers was estimated by the test of adherence to inhalers. ${ }^{6}$

The study was approved by the Research Ethics Committee of the Balearic Islands (code: IB2499/15). In addition, an independent ethics committee or institutional review board for each study center approved the final protocol.

\section{Study protocol}

All researchers were asked to recruit 12 consecutive patients with chronic obstructive airway disease: four nonsmoking asthmatics, four with COPD, two smoking asthmatics, and two with COPD with eosinophilia. The recruitment period was between October 2015 and March 2016. The flowchart of recruitment according to according to the STROBE statement (STrengthening the Reporting of OBservational studies in Epidemiology) has been published elsewere. ${ }^{5}$

The study was conducted in a single visit in which the researchers obtained and recorded information in an electronic clinical research database: anthropometric data; smoking history; a previous history of atopy, rhinitis, nasal polyposis, and comorbidities; exacerbations in the previous year; symptoms assessed by the validated Spanish version of the COPD assessment test and by the asthma control test; pharmacologic treatments; and the degree of compliance with inhalers estimated by the test of adherence to inhalers.

Fractional exhaled nitric oxide was measured by using a chemiluminescence analyzer (NIOX; Aerocrine, Stockholm, Sweden) at a flow rate of $50 \mathrm{~mL} / \mathrm{s}$, in accordance with the recommendations of the American Thoracic Society/the European Respiratory Society task force.

\section{Sputum induction}

Before the induction of sputum, patients inhaled $400 \mu \mathrm{g}$ of salbutamol. Briefly, the volume of the whole sputum was assessed, and an equal volume of dithioerythritol (Sigma, Munich, Germany) was added. The total cell count and viability were obtained simultaneously in a modified Neubauer counting chamber (Hausser Scientific, Horsham, PA, USA). Differential cell counts were expressed as the percentage of 
nonsquamous cells, and those with significant squamous contamination $(>80 \%)$ were excluded from the analysis.

\section{Sample size calculation}

An a priori sample size was calculated considering a statistical power of $80 \%$ and $5 \%$ of alpha error. A minimum of 100 asthma-COPD overlap (ACO) patients and 200 other chronic airway disease patients would be enough in order to identify statistically significant differences of $\geq 15 \%$ when compared with any of the reference groups, in most variables ranging from $10 \%$ up to $80 \%$ in $\mathrm{ACO}$.

\section{Definitions}

\section{Asthma}

Asthma with chronic airflow obstruction (post-bronchodilator $\mathrm{FEV}_{1} / \mathrm{FVC}<0.70$ ) was diagnosed according to the international guidelines, ${ }^{7}$ and they were either never-smokers or ex-smokers with a smoking history of $\leq 10$ pack-years.

\section{COPD}

COPD was diagnosed according to international recommendations ${ }^{3}$ by the presence of post-bronchodilator $\mathrm{FEV}_{1} /$ FVC $<0.70$ in patients with a smoking history of $\geq 10$ packyears in the absence of a clinical suspicion for asthma.

\section{ACO}

As described in detail previously, ${ }^{5}$ we defined ACO as an umbrella term that encompasses two related - with a similar underlying mixed (Th2 and non-Th2) inflammatory pattern and chronic airflow obstruction - but distinct entities: smoking asthmatics (SA; asthma with chronic airflow obstruction and a smoking history $\geq 20$ pack-years) and COPD with eosinophilia (COPD-e; COPD patients with eosinophil count $\geq 200$ cells $/ \mu \mathrm{L}$ in blood). We selected this threshold because below this cutoff patients are unlikely to have sputum eosinophilia, according to published evidence. ${ }^{8}$

\section{COAD with Th2-high}

Patients with chronic airflow limitation demonstrated post-bronchodilator $\mathrm{FEV}_{1} / \mathrm{FVC}<0.70$ and a sign of Th2 inflammation as described previously ${ }^{5}$ (eosinophil count $\geq 300$ eosinophils $/ \mu \mathrm{L}$ in blood or $\geq 3 \%$ in sputum).

\section{COAD with Th2-low}

Patients with chronic airflow limitation demonstrated post-bronchodilator $\mathrm{FEV}_{1} / \mathrm{FVC}<0.70$ and no signs of Th-2 inflammation as described previously ${ }^{5}$ (eosinophil count $\leq 300$ eosinophils $/ \mu \mathrm{L}$ in blood and $\leq 3 \%$ in sputum).

\section{Th2/Th I ratio}

The ratios IL-13/IL-8 or IL-5/IL-8 were calculated to detect a predominant $\mathrm{Th} 2$ profile.

\section{Measurements}

More detailed information about data management, pulmonary function tests, fractional exhaled nitric oxide (FeNO) measurement, and cell inflammatory counts in induced sputum can be found elsewhere. ${ }^{5}$ Periostin levels were measured by using a streptavidin-horseradish peroxidase enzyme-linked immunosorbent assay (ELISA) commercial kit (DuoSet ${ }^{\circledR}$ ELISA; R\&D Systems, Inc., Minneapolis, MN, USA), according to the manufacturer's instructions. The specificity of the periostin assay was $100 \%$, and the detection limit was $180 \mathrm{ng} / \mathrm{mL}$. In all the patients, serum periostin levels were measured in duplicate.

The concentrations of IL-5, IL-13, IL-8, IL-6, tumor necrosis factor- $\alpha$ (TNF- $\alpha$ ), and IL-17 in serum were analyzed by using a human cytokine magnetic bead panel (Merck Millipore, Billerica, MA, USA) according to the manufacturer's instructions. Assay sensitivity according to the manufacturer was as follows: $0.23 \mathrm{pg} / \mathrm{mL}$ for IL-13, $0.33 \mathrm{pg} / \mathrm{mL}$ for IL-17, $0.12 \mathrm{pg} / \mathrm{mL}$ for IL-5, $0.11 \mathrm{pg} / \mathrm{mL}$ for IL-6, $0.13 \mathrm{pg} / \mathrm{mL}$ for IL-8, and $0.16 \mathrm{pg} / \mathrm{mL}$ for TNF- $\alpha$.

\section{Network analysis}

A network layout of the systemic inflammatory pattern (inflammome) in the three groups of participants was built following a previously validated method. ${ }^{9}$ Each node of the network represents one biomarker, its size being proportional to the percentage of the median in reference to maximal values for each variable. Nodes are linked if a statistically significant correlation (Spearman $p<0.05$ ) was found.

\section{Statistical analysis}

Data are expressed as mean (SD) for continuous normally distributed variables: univariate pair-wise comparisons were performed by using Student's $t$-tests, while one-way analysis of variance was used to compare more than two groups (Bonferroni correction was applied for pair-wise post hoc tests). Continuous variables with non-normal distributions were expressed as median (interquartile range [P25-P75]), and comparisons among groups were carried out by using the Mann-Whitney $U$-test or Kruskal-Wallis test with the Nemenyi post hoc test. For categorical variables, counts and percentages were given in each category, and groups were compared by means of $\chi^{2}$ tests, with Bonferroni correction 
for pair-wise comparisons. Correlations between continuous variables were estimated by using Spearman test.

To explore the association of biomarkers with the COAD categories (asthma, COPD, and ACO) and to assess the corresponding discrimination ability, a discriminant analysis (DA) was carried out fitting the linear combinations of biomarkers, which allowed the best classification of patients into the diagnostic groups. The results were visually summarized through a classification map, based on the DA estimates.

The reliability of biomarkers' concentration for the diagnosis of different COAD categories and Th2 profiles was assessed by estimating the corresponding receiver operating characteristic (ROC) curves with the corresponding 95\% confidence intervals. Youden index was used for the determination of optimal cutoff values of the biomarkers. Sensitivity and specificity were also estimated for each cutoff.

All statistical analyses were performed by using the SPSS software (Version 19.0; IBM Corporation, Armonk, NY, USA).

\section{Results}

\section{Population characteristics}

As described in detail previously, 292 patients with COAD were included in the study: 94 asthmatics, 89 COPD, and 109 ACO (44 SA and 65 COPD-e) patients. Figure 1 shows the flowchart of patient recruitment. Table 1 reports the demographic, clinical, and functional characteristics of the entire sample. Briefly, patients showed a moderate degree of bronchial obstruction and mild-to-moderate level of symptoms as assessed by the COPD assessment test (CAT) and asthma control test (ACT) questionnaires. The average number of exacerbations during the last 12 months was 1.0. Baseline

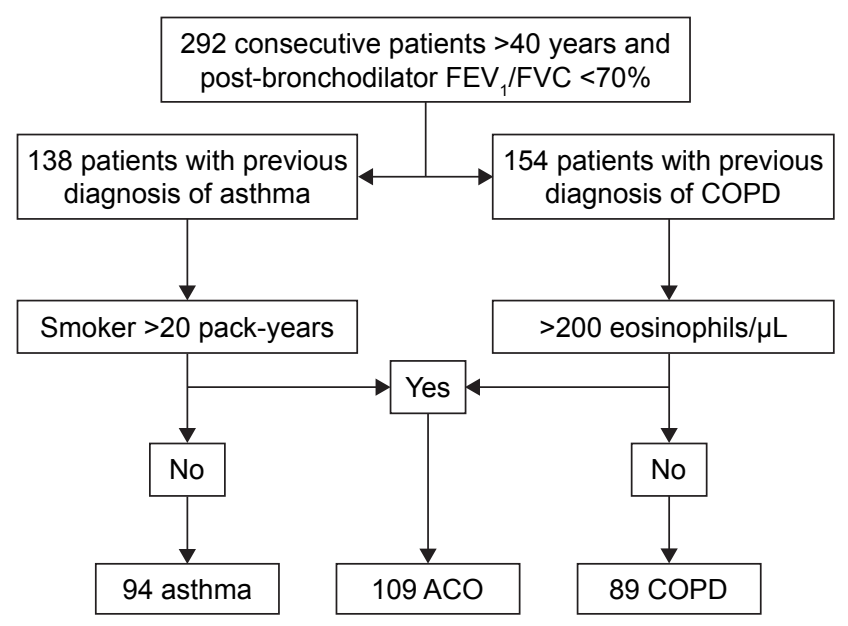

Figure I Flowchart diagram of recruitment process.

Abbreviations: ACO, asthma-COPD overlap; COPD, chronic obstructive pulmonary disease. $\mathrm{FEV}_{1}$, forced expiratory volume in the first second; FVC, forced vital capacity.
Table I Characteristics of patients according to the baseline diagnosis

\begin{tabular}{|c|c|c|c|c|}
\hline & Asthma & COPD & $\mathrm{ACO}$ & $p$-value \\
\hline $\begin{array}{l}\text { Number of } \\
\text { subjects }\end{array}$ & 94 & 89 & 109 & \\
\hline Age (years) & $61.8(12.3)$ & $67.9(8.6)$ & $63.31(10.6)$ & $<0.001$ \\
\hline Gender ( $\%$ female) & 55.3 & 24.7 & 34.9 & $<0.001$ \\
\hline BMI $\left(\mathrm{kg} / \mathrm{m}^{2}\right)$ & $28.6(4.7)$ & $28.4(4.9)$ & $28.6(6)$ & 0.96 \\
\hline Pack-years & $3.4(5.8)$ & $48.7(22.5)$ & $45.1(24)$ & $<0.00$ I \\
\hline Positive SPT (\%) & 46.8 & 7.9 & 32.1 & $<0.001$ \\
\hline Rhinitis (\%) & 53.2 & 9.3 & 31.1 & $<0.001$ \\
\hline Pre-BD FEV & $61.2(17.7)$ & $5 \mathrm{I} .4(18.2)$ & $53.8(16.5)$ & $<0.001$ \\
\hline Post-BD FEV $(\%)$ & $69.5(18.9)$ & $55.1(18.5)$ & $58.9(17)$ & $<0.001$ \\
\hline PBT (\%) & 44.6 & 19.2 & 37.7 & 0.001 \\
\hline DLCO (\%) & $83.4(18.7)$ & $63.6(25.9)$ & $66.6(19.1)$ & $<0.001$ \\
\hline $\begin{array}{l}\text { Exacerbations in } \\
\text { previous year }\end{array}$ & $0.97(1.37)$ & $1.08(1.32)$ & $0.97(1.36)$ & 0.92 \\
\hline $\begin{array}{l}\text { Blood eosinophils } \\
\text { (cells } / \mu \mathrm{L} \text { ) }\end{array}$ & $300(0,4,350)$ & $100(0,200)$ & $300(0,1,000)$ & $<0.001$ \\
\hline $\begin{array}{l}\text { Sputum eosinophils } \\
(\mathrm{n}=35, \%)\end{array}$ & $4.8(5.8)$ & $1.8(2.1)$ & $6.3(5.8)$ & 0.21 \\
\hline $\operatorname{lgE}(\mathrm{IU} / \mathrm{mL})$ & I $23(4,2,909)$ & $5 \mathrm{I}(4,2,30 \mathrm{I})$ & $89.8(4,2,500)$ & 0.094 \\
\hline $\mathrm{FeNO}(\mathrm{ppb})$ & $36.0(24.1)$ & $17.3(9.6)$ & $22.5(21)$ & $<0.001$ \\
\hline CAT & | $4.8(8.8)$ & $13.0(6.7)$ & I $3.3(7.7)$ & 0.83 \\
\hline ACT & $20.2(4.4)$ & $20.4(4.3)$ & $19.4(4.8)$ & 0.54 \\
\hline ICS (\%) & 100 & 66.3 & 75.2 & $<0.01$ \\
\hline LABA (\%) & 99 & 93.3 & 93.6 & 0.19 \\
\hline LAMA (\%) & 54.3 & 87.6 & 73.4 & $<0.01$ \\
\hline
\end{tabular}

Notes: Data are expressed as mean (SD), median (percentile 25, percentile 75 ), or $\mathrm{n}$ and \%. Overall $p$-values are reported. Exacerbations: number of severe exacerbations during the past 12 months.

Abbreviations: ACO, asthma-COPD overlap; ACT, asthma control test; BMI, body mass index; CAT, COPD assessment test; COPD, chronic obstructive pulmonary disease; DLCO, carbon monoxide diffusing capacity; FeNO, fractioned exhaled nitric oxide levels; FEV inhaled corticosteroid; IgE, immunoglobulin E; LABA, long-acting $\beta 2$-agonists; LAMA, long-acting muscarinic antagonists; PBT, positive bronchodilator test; post-BD, postbronchodilator; pre-BD, pre-bronchodilator; SPT, skin prick test.

treatment was based on long-acting $\beta_{2}$-agonist and inhaled corticosteroids (ICSs; 80\%) and long-acting muscarinic antagonist (71\%). All asthma patients, 66.3\% of COPD patients, and $75 \%$ of ACO patients were treated with ICSs. No patient from the COPD or ACO group was being treated with oral corticosteroids, whereas $6.4 \%$ of the asthmatics were taking them. Interestingly, $17.5 \%$ of asthma patients and $8.6 \%$ of ACO patients were receiving omalizumab.

\section{Cytokine and periostin values among the different diagnostic categories}

Table 2 and Figure 2 display the results of inflammatory markers measured in the three diagnostic categories. Th2related cytokines (IL-13 and IL-5) showed highest median values in asthma and lowest median values in COPD, whereas they presented intermediate results in ACO. The only statistically significant difference was in IL-5 levels between asthma 
Table 2 Biomarker values according to the baseline diagnosis

\begin{tabular}{|c|c|c|c|c|}
\hline & Asthma $(n=94)$ & COPD $(n=89)$ & $\operatorname{ACO}(n=109)$ & $p$-value \\
\hline IL-6 (pg/mL) & I.77 (0.7I-3.66) & $1.13(0.43-2.30)^{*}$ & $1.42(0.57-2.44)$ & 0.031 \\
\hline IL-8 (pg/mL) & $7.03(4.69-10.44)$ & $9.45(6.61-13.12)^{* *}$ & $9.58(6.39-13.5 \mathrm{I})^{* *}$ & $<0.001$ \\
\hline TNF- $\alpha$ (pg/mL) & $3.93(3.11-5.50)$ & $4.21(2.57-5.68)$ & $4.17(3.22-5.43)$ & 0.660 \\
\hline IL-I3 (pg/mL) & $2.98(0.23-7.63)$ & $\mathrm{I} .25(0.23-5.3 \mathrm{I})$ & $1.98(0.23-9.92)$ & 0.213 \\
\hline IL-5 (pg/mL) & $2.09(0.6 \mathrm{I}-3.57)$ & I.II $(0.12-2.42)^{*}$ & $\mathrm{I} .73(0.4 \mathrm{I}-2.77)$ & 0.012 \\
\hline Periostin (ng/mL) & $37.4(26.6-49.2)$ & $33.5(27.7-49.9)$ & $35(27.9-44)$ & 0.232 \\
\hline IL-I7 (pg/mL) & $6.94(3.11-15.90)$ & $5.33(1.4 I-11.37)$ & $6.75(2.64-12.47)$ & 0.075 \\
\hline IL-I3/IL-8 & $0.43(0.05-0.63)$ & $0.15(0.04-0.56)$ & $0.14(0.03-0.53)$ & 0.013 \\
\hline IL-5/IL-8 & $0.25(0.08-0.63)$ & $0.12(0.02-0.25)$ & $0.14(0.05-0.29)$ & $<0.001$ \\
\hline FeNO (ppb) & $33(|9.8-4| .3)$ & $17.3(12-18.8)^{* *}$ & $19.6(10-24.6)^{* *}$ & $<0.001$ \\
\hline
\end{tabular}

Notes: Overall $p$-values from Kruskal-Wallis ANOVA; pairwise $p$-values from Mann-Whitney $U$-test, Bonferroni-corrected: $* p<0.05$ vs asthma; $* * p<0.00$ I vs asthma. Abbreviations: ACO, asthma-COPD overlap; ANOVA, analysis of variance; COPD, chronic obstructive pulmonary disease; FeNO, fractional exhaled nitric oxide; TNF- $\alpha$, tumor necrosis factor- $\alpha$.

and COPD (2.09 vs $1.11 \mathrm{pg} / \mathrm{mL} ; p=0.03)$. IL-17 and TNF- $\alpha$ levels were similar between the groups. Periostin levels were not different between the three categories. Non-Th2-related cytokines such as IL-8 were found to be increased in COPD and ACO compared with asthma $(p<0.001)$.

Th2/Th1 ratios (IL-5/IL-8 and IL-5/IL-13) were higher in asthma ( 0.25 and 0.43 , respectively) than in the other categories without differences between $\mathrm{ACO}(0.14$ and 0.14$)$ and COPD (0.12 and 0.15).

\section{Discriminant and network analysis}

Overall, the correlation between the cytokines was poor. It is worth emphasizing that correlation between IL-5 and IL-13 was $0.489(p<0.0001)$, and IL-17 correlated moderately with IL-5 ( $\mathrm{r}=0.553 ; p<0.0001)$, IL-13 ( $\mathrm{r}=0.550 ; p<0.0001)$, and IL-6 ( $\mathrm{r}=0.579 ; p<0.0001)$. IL-8 and TNF- $\alpha$ showed a weak correlation between them $(\mathrm{r}=0.207 ; p<0.0001)$ and

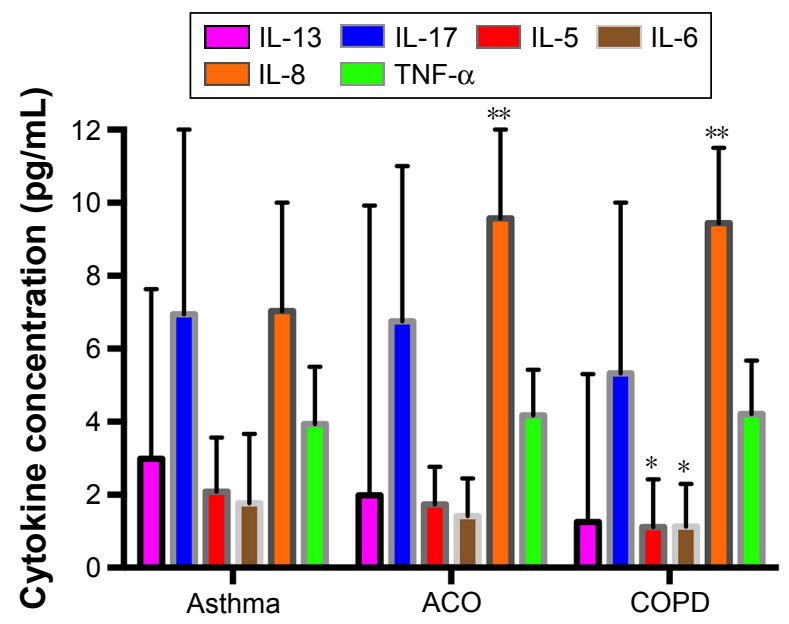

Figure 2 Cytokine concentrations in asthma, ACO, and COPD.

Notes: $* p<0.05$ vs asthma; $* * p<0.001$ vs asthma.

Abbreviations: ACO, asthma-COPD overlap; COPD, chronic obstructive pulmonary disease; TNF- $\alpha$, tumor necrosis factor- $\alpha$. no statistically significant correlation with IL-5, IL-13, and IL-17. IL-6 correlated moderately with the other cytokines. Blood eosinophils correlated weakly with IL-5 ( $\mathrm{r}=0.122$; $p=0.40)$ and with periostin $(\mathrm{r}=0.211 ; p<0.0001)$. Periostin did not correlate significantly with blood cytokines.

Two principal components (linear combination of markers), explaining about $60 \%$ of the total variance in the data, were identified. The first discriminant score (DS1) was mainly characterized by the values of IL-5, IL-13, IL-6, and TNF- $\alpha$, while IL- 8 had more weight in the second discriminant score (DS2). Figure 3 shows the scatterplot of individual

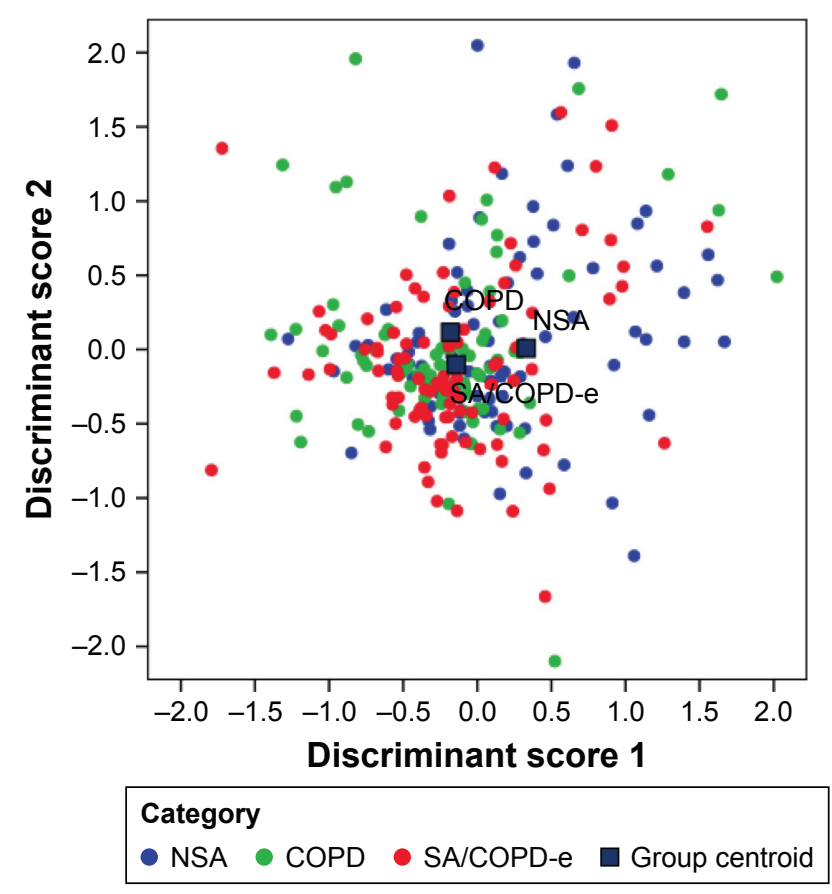

Figure 3 Scatterplot of the combination of values from discriminant scores I and 2 as compared to each patient's COAD category.

Abbreviations: COAD, chronic obstructive airway disease; COPD, chronic obstructive pulmonary disease; COPD-e, COPD with eosinophilia; NSA, nonsmoking asthmatics; SA, smoking asthmatics. 
values computed for each patient based on DS1 and DS2, identifying the patient's diagnostic category (asthma, COPD, and ACO). Patients from different categories had very similar DS values, so that a clear and distinct pattern could not be observed in cytokine relationship. Less than one half of the patients $(42.8 \%)$ were correctly classified by the two estimated DSs, confirming the limited discrimination abilities of these markers.

The network representation of the weight and interaction of inflammatory markers (inflammome) in the three categories yielded two different scenarios, being IL-13 and IL-17 the most connected nodes but with different weights of the nodes, showing IL-5 and IL-8 with the most marked differences in node weight. The inflammome of ACO was a mixture of the two previous networks, being IL-5 and IL-8 equally important and with IL-13 and IL-17 still in the middle of the network (Figure 4).

\section{Diagnostic accuracy of the potential biomarkers for the identification of different diagnostic categories or Th2-high and Th2-low inflammatory phenotypes}

Neither a single cytokine nor Th2/Th1 ratios yielded enough sensitivity and specificity to accurately identify the various diagnostic categories of COAD (asthma, COPD, and ACO). The area under the ROC curve (AUC) was $<0.7$ in every case. Table S1 displays the AUCs for each cytokine or periostin and the diagnostic categories of COAD.

Some cytokines were more highly expressed in the Th2high group compared with the Th2-low group (Table 3), medians (interquartile ranges): IL-5 (1.96 [0.87-3.34] vs 1.51 [0.29-2.88] pg/mL; $p=0.025)$, TNF- $\alpha$ (4.32 [3.20-6.16] vs 3.95 [2.91-5.32] pg/mL; $p=0.055)$, and periostin $(39.73$ [28.05-54.40] vs 34.20 [26.94-41.43] ng/mL; $p=0.005)$.
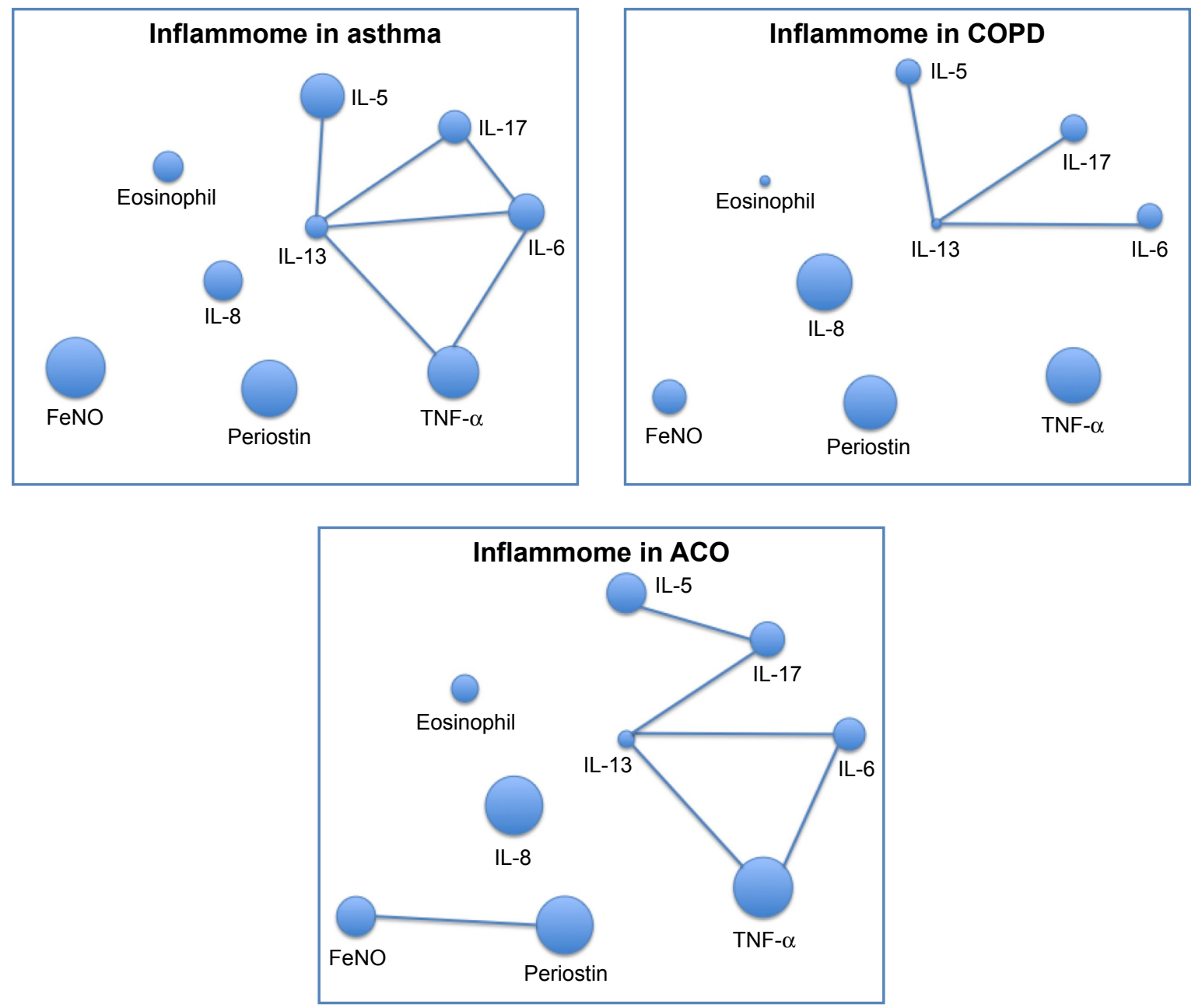

Figure 4 Network layout of inflammome in asthma, COPD, and ACO.

Abbreviations: ACO, asthma-COPD overlap; COPD, chronic obstructive pulmonary disease; FeNO, fractional exhaled nitric oxide; TNF- $\alpha$, tumor necrosis factor- $\alpha$. 
Table 3 Blood biomarkers in Th2-high and Th2-low patients

\begin{tabular}{llll}
\hline & $\begin{array}{l}\text { Th2-high pattern } \\
(\mathbf{n}=94)\end{array}$ & $\begin{array}{l}\text { Th2-low pattern } \\
(\mathbf{n}=1 \mathbf{1 9 7})\end{array}$ & p-value \\
\hline $\mathrm{IL}-6(\mathrm{pg} / \mathrm{mL})$ & $\mathrm{I} .75(0.58-2.67)$ & $\mathrm{I} .3 \mathrm{I}(0.50-2.63)$ & 0.174 \\
$\mathrm{IL}-8(\mathrm{pg} / \mathrm{mL})$ & $8.34(5.37-12.77)$ & $8.40(5.88-12.77)$ & 0.856 \\
$\mathrm{TNF}-\alpha(\mathrm{pg} / \mathrm{mL})$ & $4.32(3.20-6.16)$ & $3.95(2.91-5.32)$ & 0.055 \\
$\mathrm{IL}-\mathrm{I3}(\mathrm{pg} / \mathrm{mL})$ & $2.92(0.26-5.59)$ & $\mathrm{I} .65(0.23-5.58)$ & 0.150 \\
$\mathrm{IL}-5(\mathrm{pg} / \mathrm{mL})$ & $\mathrm{I} .96(0.87-3.34)$ & $1.51(0.29-2.88)$ & 0.025 \\
Periostin $(\mathrm{ng} / \mathrm{mL})$ & $39.73(28.05-54.40)$ & $34.20(26.94-41.43)$ & 0.005 \\
$\mathrm{IL}-13 / \mathrm{IL}-8$ & $0.26(0.06-0.66)$ & $0.15(0.03-0.78)$ & 0.182 \\
$\mathrm{IL}-5 / \mathrm{IL}-8$ & $0.23(0.08-0.40)$ & $0.13(0.03-0.33)$ & 0.019 \\
$\mathrm{IL}-17(\mathrm{pg} / \mathrm{mL})$ & $7.37(3.46-13.76)$ & $5.73(2.10-12.12)$ & 0.125 \\
\hline
\end{tabular}

Notes: Data are expressed as medians and interquartile ranges; $p$-values from the Mann-Whitney U-test.

Abbreviation: TNF- $\alpha$, tumor necrosis factor- $\alpha$.

No statistically significant differences were found between the two groups in the other analyzed cytokines, alone or in combination (Table 3).

Again, since the AUC was $<0.7$ in every case, neither a single cytokine nor periostin, nor any combination of them, yielded enough sensitivity and specificity to accurately predict the Th2-high and Th2-low molecular phenotype. A cutoff value of FeNO $>17 \mathrm{ppb}$ showed better AUC $(0.707$ [0.642-0.772], $p<0.001)$ than the cytokines or periostin in blood (Table 4).

\section{Association of cytokines or periostin with clinical outcomes}

The values of the different cytokines or periostin (alone or in combination) were not different between the patients

Table 4 Biomarker ROC and best cutoff point to predict Th2high or Th2-low patterns

\begin{tabular}{lll}
\hline Biomarker & ROC & Value \\
\hline IL-6 & AUC $(95 \% \mathrm{Cl})$ & $0.550(0.479-0.620)$ \\
& Best cutoff $(\mathrm{pg} / \mathrm{mL})$ & NA \\
IL-8 & AUC $(95 \% \mathrm{Cl})$ & $0.507(0.434-0.579)$ \\
& Best cutoff $(\mathrm{pg} / \mathrm{mL})$ & NA \\
TNF- $\alpha$ & AUC $(95 \% \mathrm{Cl})$ & $0.570(0.499-0.64 \mathrm{I})$ \\
& Best cutoff $(\mathrm{pg} / \mathrm{mL})$ & 4.04 \\
$\mathrm{IL}-13$ & AUC $(95 \% \mathrm{Cl})$ & $0.552(0.482-0.623)$ \\
& Best cutoff $(\mathrm{pg} / \mathrm{mL})$ & NA \\
IL-5 & AUC $(95 \% \mathrm{Cl})$ & $0.582(0.5 \mathrm{I} 2-0.652)$ \\
& Best cutoff $(\mathrm{pg} / \mathrm{mL})$ & 1.79 \\
Periostin & AUC $(95 \% \mathrm{Cl})$ & $0.602(0.528-0.677)$ \\
& Best cutoff $(\mathrm{IU} / \mathrm{mL})$ & 35.2 \\
$\mathrm{IL}-5+\mathrm{IL}-13$ & AUC $(95 \% \mathrm{Cl})$ & $0.579(0.508-0.650)$ \\
$\mathrm{IL}-5+\mathrm{IL}-\mathrm{I3}+\mathrm{IL}-8$ & AUC $(95 \% \mathrm{Cl})$ & $0.593(0.523-0.662)$ \\
$\mathrm{IL}-13 / \mathrm{IL}-8$ & AUC $(95 \% \mathrm{Cl})$ & $0.549(0.479-0.619)$ \\
$\mathrm{IL}-5 / \mathrm{IL}-8$ & AUC $(95 \% \mathrm{Cl})$ & $0.586(0.5 \mathrm{I}-0.654)$ \\
& Best cutoff & 0.15 \\
$\mathrm{IL}-17$ & AUC $(95 \% \mathrm{Cl})$ & $0.556(0.486-0.626)$ \\
& Best cutoff $(\mathrm{pg} / \mathrm{mL})$ & NA \\
\hline
\end{tabular}

Abbreviations: $\mathrm{AUC}$, area under the ROC; $\mathrm{Cl}$, confidence interval; NA, not applicable; ROC, receiver operating curve; TNF- $\alpha$, tumor necrosis factor- $\alpha$. with ( $\mathrm{n}=144)$ and without $(\mathrm{n}=148)$ exacerbations, between poorly controlled $(\mathrm{ACT}<20[\mathrm{n}=85])$ and well-controlled asthmatics (ACT $\geq 20[\mathrm{n}=137]$ ), between symptomatic $(\mathrm{CAT} \geq 10[\mathrm{n}=163])$ and poorly symptomatic COPD patients (CAT $<10[\mathrm{n}=81]$ ), and between those with $\mathrm{FEV}_{1}$ above the whole population median $(1.505 \mathrm{~mL})$ and those with $\mathrm{FEV}_{1}$ below the median.

\section{Discussion}

In this study, we have found that the different inflammatory mechanisms involved in asthma and COPD may overlap in certain patients, leading to a mixture of Th2 and non-Th2 cytokine expression. ACO shows therefore a heterogeneous inflammatory process that overlaps the characteristics of both conditions: asthma and COPD; therefore, it cannot be considered a specific phenotype of any of them. As expected, Th2-related cytokines (IL-13 and IL-5) exhibited highest median values in asthma and lowest median values in COPD with intermediate results in ACO. Inversely, IL-8 was found to be significantly increased in patients with a smoking history (COPD and ACO) in comparison with asthma. Periostin levels were not useful to distinguish between categories, but they were increased in those with a more predictable "Th2high signature" (asthma and ACO) with respect to COPD. Interestingly, IL-13 was the most connected node of the inflammome in the three conditions. Taken together, these findings are congruent with the heterogeneous inflammatory background of COAD patients, probably a continuum between non-Th2 and Th2 inflammation with substantial overlap in a high proportion of cases.

Since several biologic therapies are now being tested in asthma and COPD, the identification of biological markers reflecting the underlying disease process seems to be of particular relevance to implementing more personalized approaches to therapeutic decisions in airway disease. However, to date, very few studies have addressed the underlying mechanisms of inflammation in ACO. Kitaguchi et al, ${ }^{10}$ in a retrospective study with a small sample size, found that COPD patients with asthmatic symptoms had high peripheral and sputum eosinophil counts and better reversibility response to treatment with ICSs. On the other hand, Gao et al ${ }^{11}$ aimed at finding sputum biomarkers capable of differentiating between ACO, COPD, and asthma patients. They found that only sputum neutrophil gelatinase-associated lipocalin levels were significantly increased in patients with ACO in comparison with the asthma and COPD groups. In this study, sputum IL-13 was increased in asthma, COPD, and ACO in comparison with healthy and smoker controls, which could 
be sustaining the central role in the inflammome we found in our study. However, since the definitions of the different diagnostic categories are somewhat arbitrary and based on nonspecific clinical features, the design of this study was subjected to the same limitations as all the previously published studies on this topic. In theory, it would be expected to find some evidence of Th1 inflammation (characteristic, but not pathognomonic of COPD) and some evidence of Th2 inflammation (characteristic, but not pathognomonic of asthma) in patients with ACO. ${ }^{12}$ Unfortunately, there is a lack of reliable Th1 biomarkers; therefore, we have to rely on the assumption that individuals with a smoking history and "fixed bronchial obstruction" who show some evidence of Th2 inflammation can accurately be classified as ACO. ${ }^{13}$ Our finding of intermediate IL-5 and IL-13 levels in ACO categories, when compared to asthma and COPD, could give some support to this hypothesis.

Based on DA results, we were unable to find meaningful clusters of association between the different biomarkers, and in consequence, we were not able to demonstrate a link between the three clinical categories of COAD (asthma, COPD, and ACO) with clearly defined different inflammatory patterns. However, a network representation of the interaction between the inflammatory markers yielded two clearly different networks that overlap in ACO. This supports the hypothesis that inflammatory pattern observed in ACO is a blend of COPD and asthma components.

Overall, the correlation between biomarkers was poor. The weak correlation found between blood eosinophils and Th2-high cytokines (IL-5 and IL-13) was of particular interest because it contradicts the results obtained by Agache et al, ${ }^{14}$ who observed an almost perfect $(r>0.90)$ association between these variables in 64 adult asthmatics. Our results were not different when we analyzed separately the correlation of these biomarkers in the asthma group, although it should be taken into account that all of our patients showed "fixed bronchial obstruction," whereas this feature was only present in 6\% of Agache et al's sample, which might reflect notable differences in the studied populations.

Two reasons could help to explain the observed lack of association between clinical categories and putative biomarkers. First, it has been reported that asthma and COPD demonstrate a significant overlap in the airway cytokine profile, which suggests the coexistence of different phenotypes of COAD sharing a common cytokine network. ${ }^{15,16}$ The central role of IL-13 and IL-17 in the network would be in keeping with this result. Moreover, we should bear in mind that asthma and COPD are themselves heterogeneous diseases with various inflammatory phenotypes. Solleiro-Villavicencio et $\mathrm{al}^{17}$ observed that the inflammatory response induced by the exposure to biomass smoke differs greatly from that caused by tobacco smoking, and Khurana et $\mathrm{al}^{18}$ found that "COPD persistent sputum producers" have increased concentrations of some inflammatory mediators in the airways when compared to nonpersistent sputum producers. On the other hand, Seys et $\mathrm{al}^{19}$ found five different asthma clusters based on sputum cytokine expression, which underscores the heterogeneity in airway inflammation among different asthma patients. Taken together, these studies point out a patient-to-patient variability of the inflammatory markers in individuals with COAD. Second, there seems to be no clear correlation between biomarkers in systemic and pulmonary compartments. Although serum cytokines have shown sufficient reliability and repeatability over time, several authors observed only few and weak associations between lung and serum biomarkers, both in COPD ${ }^{20,21}$ and asthma patients. ${ }^{22}$ Thus, it is possible that measuring serum cytokines does not sufficiently reflect the inflammatory processes within the airways.

As described in detail previously, ${ }^{5}$ "our work has several limitations that deserve a comment. First, we used arbitrary cutoff points of blood eosinophil to recruit patients and to define Th2 profile, but we used the available published information that supports this view. However, we were more stringent in the definition of Th2, using a cutoff of 300 cells $/ \mu \mathrm{L}$, which is widely accepted. ${ }^{23}$ In addition, a large number of patients were receiving ICSs, and some of them were being treated with oral corticosteroids or omalizumab, which could have an effect masking differences between groups and modifying the expression of Th2 biomarkers such as blood/ sputum eosinophils and serum cytokines. Third, the limit of 20 pack-years of smoking exposure in asthmatics could be too artificially high. However, by doing so, we make sure that this phenotype is going to overlap the characteristics of smoking-related inflammation on the top of the baseline asthmatic inflammation."

\section{Conclusion}

The heterogeneity of asthma and COPD induces different inflammatory pathways that coexist in ACO, being IL-13 the common node orchestrating the inflammome in these conditions. Nevertheless, neither non-Th2-related nor Th2-related serum cytokines or periostin (alone or combined) permitted to distinguish between COAD categories or to predict a Th2high inflammatory pattern. 


\section{Acknowledgments}

The authors are grateful to all the patients who participated in the study. A number of investigators contributed to the study logistics and they are listed in the Supplementary materials. The project was endorsed by the COPD and Asthma Research Board (PII de EPOC y asma) of the Spanish Society of Pneumology and Thoracic Surgery (SEPAR). The project was partially funded by the Fondo de Investigaciones Sanitarias, Instituto de Salud Carlos III, Ministerio de Economia y Competitividad (FIS 15/01263) through European Union FEDER funds and by unrestricted grant from Chiesi España S.A.U.

\section{Author contributions}

All the authors made substantial contributions to conception and design, acquisition of data, or analysis and interpretation of data; drafted the article and revised it critically for important intellectual content; gave the final approval of the version to be published; and agreed to be accountable for all aspects of the work in ensuring that questions related to the accuracy or integrity of any part of the work are appropriately investigated and resolved. More specifically, BGC and LPL designed the study, recruited patients, analyzed the results, and wrote the manuscript. AI, NdC, and CC performed the cytokine analysis. ALV, AT, JLLC, JBS, EMM, JLI, IB, VP, MM, and JJSC recruited patients and reviewed the manuscript. The rest of the CHACOS study team recruited patients.

\section{Disclosure}

Dr Pérez de Llano reports grants from SEPAR, during the conduct of the study, and grants and personal fees from Novartis, Chiesi, AstraZeneca, and Pfizer, outside the submitted work. Dr Cosío reports grants from SEPAR, during the conduct of the study, and personal fees from AstraZeneca, grants from Boehringer Ingelheim, grants and personal fees from Novartis, Chiesi, Rovi, Menarini, and Esteve, outside the submitted work. Dr Izquierdo reports and has received funds for advisory boards and lectures from Almirall, AstraZeneca, Bayer, GlaxoSmithKline, Novartis, Pfizer, Boehringer Ingelheim, Takeda, Chiesi, and Menarini. Dr Soler-Cataluña reports grants from SEPAR, during the conduct of the study and grants and personal fees from AstraZeneca, Boehringer Ingelheim, Novartis, Chiesi, Rovi, Menarini, and Esteve, outside the submitted work. Dr Miravitlles reports personal fees from Almirall, Boehringer Ingelheim, AstraZeneca, Chiesi, GlaxoSmithKline, Menarini, Teva, Grifols, and Novartis, outside the submitted work. The authors report no other conflicts of interest in this work.

\section{References}

1. Barnes PJ. Immunology of asthma and chronic obstructive pulmonary disease. Nat Rev Immunol. 2008;8(3):183-192.

2. Orie NG, Slutter HJ, de Vries, Tammeling GJ. [Chronic nonspecific respiratory diseases]. Ned Tijdschr Geneeskd. 1961;105:2136-2139. Dutch.

3. Vogelmeier CF, Criner GJ, Martinez FJ, et al. Global Strategy for the Diagnosis, Management, and Prevention of Chronic Obstructive Lung Disease 2017 Report: GOLD Executive Summary. Arch Bronconeumol. 2017;53(3):128-149. English, Spanish.

4. Cosio BG, Soriano JB, Lopez-Campos JL, et al. Defining the asthma-COPD overlap syndrome in a COPD cohort. Chest. 2016; 149(1):45-52.

5. Cosio BG, Pérez de Llano L, Lopez Viña A, et al. Th-2 signature in chronic airway diseases: towards the extinction of asthma-COPD overlap syndrome? Eur Respir J. 2017;49(5):pii:1602397.

6. Plaza V, Fernandez-Rodriguez C, Melero C, et al. Validation of the 'Test of the Adherence to Inhalers' (TAI) for asthma and COPD patients. J Aerosol Med Pulm Drug Deliv. 2016;29(2):142-152.

7. Global Initiative for Asthma (GINA). Global Strategy for Asthma Management and Prevention; updated 2017. Available from: http://ginasthma. org/2017-gina-report-global-strategy-for-asthma-management-andprevention/ Chapter 1, page 14. Accessed 17 January 17, 2018.

8. Negewo NA, McDonald VM, Baines KJ, et al. Peripheral blood eosinophils: a surrogate marker for airway eosinophilia in stable COPD. Int J Chron Obstruct Pulmon Dis. 2016;11:1495-1504.

9. Agusti A, Edwards LD, Rennard SI, et al. Persistent systemic inflammation is associated with poor clinical outcomes in COPD: a novel phenotype. PLoS One. 2012;7(5):e37483.

10. Kitaguchi Y, Komatsu Y, Fujimoto K, Hanaoka M, Kubo K. Sputum eosinophilia can predict responsiveness to inhaled corticosteroid treatment in patients with overlap syndrome of COPD and asthma. Int $J$ Chron Obstruct Pulmon Dis. 2012;7:283-289.

11. Gao J, Iwamoto H, Koskela J, et al. Characterization of sputum biomarkers for asthma-COPD overlap syndrome. Int J Chron Obstruct Pulmon Dis. 2016;11:2457-2465.

12. Pérez de Llano LA, Cosio BG. Asthma, chronic obstructive pulmonary disease and other combinations. Arch Bronconeumol. 2016;52(10): 499-500. English, Spanish.

13. Miravitlles M, Alvarez-Gutierrez FJ, Calle M, et al. Algorithm for identification of asthma-COPD overlap: consensus between the Spanish COPD and asthma guidelines. Eur Respir J. 2017;49(5):pii: 1700068

14. Agache I, Strasser DS, Klenk A, et al. Serum IL-5 and IL-13 consistently serve as the best predictors for the blood eosinophilia phenotype in adult asthmatics. Allergy. 2016;71(8):1192-1202.

15. Ghebre MA, Bafadhel M, Desai D, et al. Biological clustering supports both "Dutch" and "British" hypotheses of asthma and chronic obstructive pulmonary disease. J Allergy Clin Immunol. 2015;135(1):63-72.

16. Paplinska-Goryca M, Nejman-Gryz P, Gorska K, Bialek-Gosk K, Hermanowicz-Salamon J, Krenke R. Expression of inflammatory mediators in induced sputum: comparative study in asthma and COPD. Adv Exp Med Biol. Epub 2016 Oct 14.

17. Solleiro-Villavicencio H, Quintana-Carrillo R, Falfan-Valencia R, Vargas-Rojas MI. Chronic obstructive pulmonary disease induced by exposure to biomass smoke is associated with a Th2 cytokine production profile. Clin Immunol. 2015;161(2):150-155.

18. Khurana S, Ravi A, Sutula J, et al. Clinical characteristics and airway inflammation profile of COPD persistent sputum producers. Respir Med. 2014;108(12):1761-1770.

19. Seys SF, Scheers H, Van den Brande P, et al. Cluster analysis of sputum cytokine-high profiles reveals diversity in $\mathrm{T}(\mathrm{h}) 2$-high asthma patients. Respir Res. 2017;18(1):39.

20. Ropcke S, Holz O, Lauer G, et al. Repeatability of and relationship between potential COPD biomarkers in bronchoalveolar lavage, bronchial biopsies, serum, and induced sputum. PLoS One. 2012; 7(10):e46207. 
21. Nunez B, Sauleda J, Garcia-Aymerich J, et al. Lack of correlation between pulmonary and systemic inflammation markers in patients with chronic obstructive pulmonary disease: a simultaneous, twocompartmental analysis. Arch Bronconeumol. 2016;52(7):361-367. English, Spanish.

22. Mukherjee M, Nair P. Blood or sputum eosinophils to guide asthma therapy? Lancet Respir Med. 2015;3(11):824-825.
23. Christenson SA, Steiling K, van den Berge M, et al. Asthma-COPD overlap. Clinical relevance of genomic signatures of type 2 inflammation in chronic obstructive pulmonary disease. Am J Respir Crit Care Med. 2015;191(7):758-766 


\section{Supplementary materials \\ Authorship}

Members of the CHACOS Study Group

DUE Rocio Cordova, Hospital Universitario Son EspasesIdISPa, CIBERES, Palma de Mallorca, Spain; Dr Sergi Pascual, Dra Mireia Admetlló, Hospital del Mar, Barcelona, Spain; Dra Salud Santos, Dr Gianluca Cotta Ramusino, Hospital Universitari de Bellvitge, Barcelona, Spain; Dra Carmen Gómez Neira, Dra Irene Carla Martin Robles, Hospital Universitario Lucus Augusti, Lugo, Spain; Dr Alberto Fernández-Villar, Dra Cristina Represas, Dra Ana Priegue Carreracon Hospital Xeneral de Vigo, Vigo, Spain; Dra Andrea Trisán Alonso Hospital Puerta del Hierro, Madrid, Spain; Dr Francisco García Río, Dr Raúl Galera Martínez, Dra Raquel Casitas Mateo, Dra Elisabet Martínez Cerón, Hospital La Paz, Madrid, Spain; Dra Sagrario Mayoralas, Dr Adalberto Pacheco Galván, Hospital Universitario Ramón y Cajal, Madrid, Spain; Dr Luis Puente Maestu, Dra Julia Garcia de Pedro, Dra Pilar Sanz, Hospital General Universitario Gregorio Marañón, Madrid, Spain; Dr José Belda Ramírez, Dr Fernando Sánchez-Toril López, DrRafael Peris Cardells, Hospital Arnau de Vilanova, Valencia, Spain; Dra Maria Climent Gregori, Hospital Universitario Dr Peset, Valencia, Spain; Dra Patricia Garcia-Sidro, Hospital Castellón de la Plana, Castellón, Spain; Dra Cleofe Fernández, Dra Maria Encarnación Barroso Medel, Hospital General Universitario de Alicante, Alicante, Spain; Dra María Abad Arranz, Dr Eduardo Márquez Martín, Dr Francisco Ortega, Ruiz Hospital Virgen del Rocío, Sevilla, Spain; Dr Bernardino Alcazar, Hospital Alta Resolución de la Loja, Granada, Spain; Dr Javier Calleja, Raúl Godoy Mayoral, Javier Cruz Ruiz, Hospital General Universitario de Albacete, Albacete, Spain; Dra Nuria Marina Malanda,
Dr Juan Bautista Gáldiz Iturri, Dra Elena López de Santa Maria Miró, Hospital Universitario Cruces, Bilbao, Spain; Dr Juan Luis García Rivero, Dra Ruth Valerie Alain Mbessekg, Hospital de Laredo, Laredo, Spain; Dra Cristina Martinez, Dra Rosirys Mercedes Guzmán Taveras, Dra Ana Fernandez Tena, Dra Rosario Madiedo de la Llera, Dra Ángeles Encarnación Malmierca de Dios, Instituto Nacional de Silicosis, Oviedo, Spain; Dr Jose Antonio Ros Lucas, Dra M Carmen Soto Fernández, Dr Rubén Andújar Espinosa, Hospital Universitario Virgen de la Arrixaca, Murcia, Spain; Dra Míriam Barrecheguren, Dra Cristina Esquinas, Hospital Universitari Vall d'Hebrón, Barcelona, Spain.

Table SI Biomarker AUC in the ROC for the diagnosis of the different COAD categories, alone and in combination

\begin{tabular}{|c|c|c|c|}
\hline Biomarker & Asthma & COPD & $A C O$ \\
\hline IL-I3 (pg/mL) & $\begin{array}{l}0.564 \\
(0.490-0.637)\end{array}$ & $\begin{array}{l}0.532 \\
(0.458-0.605)\end{array}$ & $\begin{array}{l}0.531 \\
(0.463-0.599)\end{array}$ \\
\hline$-5(p$ & $\begin{array}{l}0.587 \\
(0.515-0.659)\end{array}$ & $\begin{array}{l}0.600 \\
(0.528-0.672)\end{array}$ & $\begin{array}{l}0.508 \\
(0.440-0.577)\end{array}$ \\
\hline IL-I7 (pg/mL) & $\begin{array}{l}0.563 \\
(0.492-0.635)\end{array}$ & $\begin{array}{l}0.578 \\
(0.503-0.653)\end{array}$ & $\begin{array}{l}0.51 \mathrm{I} \\
(0.444-0.579)\end{array}$ \\
\hline IL-6 (pg/mL) & $\begin{array}{l}0.587 \\
(0.514-0.659)\end{array}$ & $\begin{array}{l}0.578 \\
(0.506-0.650)\end{array}$ & $\begin{array}{l}0.489 \\
(0.422-0.557)\end{array}$ \\
\hline IL-8 (pg/mL) & $\begin{array}{l}0.652 \\
(0.583-0.720)\end{array}$ & $\begin{array}{l}0.567 \\
(0.497-0.637)\end{array}$ & $\begin{array}{l}0.58 \mathrm{I} \\
(0.5 \mid 3-0.649)\end{array}$ \\
\hline $\mathrm{TI}$ & $\begin{array}{l}0.506 \\
(0.435-0.577)\end{array}$ & $\begin{array}{l}0.527 \\
(0.450-0.604)\end{array}$ & $\begin{array}{l}0.530 \\
(0.462-0.597)\end{array}$ \\
\hline IL-13/ & $\begin{array}{l}0.608 \\
(0.564-0.706)\end{array}$ & $\begin{array}{l}0.551 \\
(0.479-0.623)\end{array}$ & $\begin{array}{l}0.555 \\
(0.486-0.624)\end{array}$ \\
\hline IL-5/IL & $\begin{array}{l}0.635 \\
(0.564-0.706)\end{array}$ & $\begin{array}{l}0.605 \\
(0.533-0.677)\end{array}$ & $\begin{array}{l}0.531 \\
(0.464-0.599)\end{array}$ \\
\hline Periostin (ng/mL) & $\begin{array}{l}0.558 \\
(0.483-0.632)\end{array}$ & $\begin{array}{l}0.549 \\
(0.480-0.618)\end{array}$ & $\begin{array}{l}0.573 \\
(0.505-0.64 I)\end{array}$ \\
\hline
\end{tabular}

Note: Data shown as area under the curve (confidence interval $95 \%$ ).

Abbreviations: ACO, asthma-COPD overlap; AUC, area under the ROC; COAD, chronic obstructive airway disease; COPD, chronic obstructive pulmonary disease; ROC, receiver operating curve; TNF- $\alpha$, tumor necrosis factor- $\alpha$.
International Journal of COPD

\section{Publish your work in this journal}

The International Journal of COPD is an international, peer-reviewed journal of therapeutics and pharmacology focusing on concise rapid reporting of clinical studies and reviews in COPD. Special focus is given to the pathophysiological processes underlying the disease, intervention programs, patient focused education, and self management protocols.

\section{Dovepress}

This journal is indexed on PubMed Central, MedLine and CAS. The manuscript management system is completely online and includes a very quick and fair peer-review system, which is all easy to use. Visit $\mathrm{http}: / / \mathrm{www}$.dovepress.com/testimonials.php to read real quotes from published authors. 